\title{
Hartman-Wintner type inequalities for a class of fractional BVPs with higher order
}

Jackie Harjani ${ }^{1 *} \mathbb{D}$, Kishin Sadarangani ${ }^{1}$ and Bessem Samet ${ }^{2}$

"Correspondence:

jackie.harjani@ulpgc.es

1 Departamento de Matemáticas, Universidad de Las Palmas de Gran Canaria, Las Palmas de Gran Canaria, Spain

Full list of author information is available at the end of the article

\begin{abstract}
In this paper, we derive some Hartman-Wintner type inequalities for a certain higher order fractional boundary value problem. As an application of our results, we obtain a lower bound for the eigenvalues of the corresponding fractional operator.
\end{abstract}

MSC: 26A33; 34A08; 34K37

Keywords: Hartman-Wintner inequality; Lyapunov inequality; Fractional boundary value problem; Higher order; Eigenvalue

\section{Introduction and preliminaries}

Consider the following boundary value problem with Dirichlet conditions:

$$
\left\{\begin{array}{l}
x^{\prime \prime}(t)+q(t) x(t)=0, \quad a<t<b, \\
x(a)=x(b)=0,
\end{array}\right.
$$

where $q:[a, b] \rightarrow \mathbb{R}$ is a continuous function. Lyapunov [26] proved that if the abovementioned boundary value problem has a nontrivial solution, then

$$
\int_{a}^{b}|q(t)| d t>\frac{4}{b-a}
$$

and the constant 4 is sharp, which means that it cannot be replaced by a larger number. Inequality (1.2) has found many applications in the study of different properties of the solutions of (1.1). Due to its usefulness in various applications, several generalizations and extensions of Lyapunov inequality have been given by various authors. In particular, Hartman and Wintner [15] proved that if (1.1) has a nontrivial solution, then

$$
\int_{a}^{b}(b-s)(s-a) q^{+}(s) d s>b-a,
$$

where

$$
q^{+}(s)=\max \{q(s), 0\}, \quad s \in[a, b] .
$$

For other generalizations and extensions of Lyapunov's inequality, we refer the reader to $[5,7,10,14,19,29,30,34]$ and the references therein.

(c) The Author(s) 2019. This article is distributed under the terms of the Creative Commons Attribution 4.0 International License (http://creativecommons.org/licenses/by/4.0/), which permits unrestricted use, distribution, and reproduction in any medium, provided you give appropriate credit to the original author(s) and the source, provide a link to the Creative Commons license, and indicate if changes were made. 
In recent years, fractional calculus is becoming very popular among various fields due to its widely applications in science and engineering (see $[1-4,8,9,16,18,24,27]$ and the references therein). Due to this fact, different Lyapunov type inequalities have been obtained recently for various fractional boundary value problems. The first work in this direction is the paper [11] of Ferreira, where he established a Lyapunov type inequality for the fractional boundary value problem

$$
\left\{\begin{array}{l}
D_{a}^{\alpha} x(t)+q(t) x(t)=0, \quad a<t<b, \\
x(a)=x(b)=0,
\end{array}\right.
$$

where $1<\alpha<2, q:[a, b] \rightarrow \mathbb{R}$ is a continuous function and $D_{a}^{\alpha}$ is the Riemann-Liouville fractional derivative of order $\alpha$. Ferreira proved that if the above fractional boundary value problem has a nontrivial solution, then

$$
\int_{a}^{b}|q(t)| d t>\Gamma(\alpha)\left(\frac{4}{b-a}\right)^{\alpha-1}
$$

Passing to the limit as $\alpha \rightarrow 2^{-}$in inequality (1.3), one obtains Lyapunov's classical inequality (1.2). In [12], Ferreira derived a Lyapunov type inequality for the Caputo fractional boundary value problem

$$
\left\{\begin{array}{l}
{ }^{C} D_{a}^{\alpha} x(t)+q(t) x(t)=0, \quad a<t<b, \\
x(a)=x(b)=0,
\end{array}\right.
$$

where $1<\alpha<2, q:[a, b] \rightarrow \mathbb{R}$ is a continuous function and ${ }^{C} D_{a}^{\alpha}$ is the Caputo fractional derivative of order $\alpha$. He proved that if the above fractional boundary value problem admits a nontrivial solution, then

$$
\int_{a}^{b}|q(t)| d t>\frac{\Gamma(\alpha) \alpha^{\alpha}}{[(\alpha-1)(b-a)]^{\alpha-1}}
$$

Similarly, passing to the limit as $\alpha \rightarrow 2^{-}$in inequality (1.4), one obtains Lyapunov's classical inequality (1.2). Moreover, a nice application on the zeros of a certain Mittag-Leffler function was presented in [12]. Motivated by the above cited work, some authors continued the study of Lyapunov type inequalities for different fractional boundary value problems. We refer to Jleli and Samet [21-23], Rong and Bai [32], O’Regan and Samet [28], Cabrera et al. [6] and the references therein.

In this paper, we are concerned with the problem of finding some Hartman-Wintner type inequalities for the following higher order fractional boundary value problem:

$$
\left\{\begin{array}{l}
D_{a}^{v} x(t)+q(t) x(t)=0, \quad a<t<b \\
x^{(i)}(a)=0, \quad 0 \leq i \leq n-2, \quad\left[D_{a}^{\alpha} x(t)\right]_{t=b}=0
\end{array}\right.
$$

where $D_{a}^{v}$ denotes the standard Riemann-Liouville fractional derivative of order $v, n \geq 3$, $n-1<v<n, n-3<\alpha<n-2$ and $q:[a, b] \rightarrow \mathbb{R}$ is a continuous function. Next, we present some applications to eigenvalue problems. Note that (1.5) contains as special cases various 
fractional boundary value problems that arise in nonlinear analysis and its applications. For example, for $n=4,(1.5)$ is a fractional model of an elastic beam equation (see [25]).

In the sequel and for convenience of the reader, we present some definitions and basic facts about fractional calculus which will be used later. For more details, we refer to [17, $20,24,31,33]$.

Let $(a, b) \in \mathbb{R}^{2}$ be such that $a<b$. Let $\mathbb{N}$ be the set of positive integers. We denote by $A C^{1}([a, b])$ the set of absolutely continuous functions in $[a, b]$. For $n \in \mathbb{N}$, let

$$
A C^{n}([a, b])=\left\{f \in C^{n-1}([a, b]): f^{(n-1)} \in A C^{1}([a, b])\right\} .
$$

Definition 1 Let $f \in L^{1}(a, b)$ and $\alpha>0$. The Riemann-Liouville fractional integral of order $\alpha$ of $f$ is defined as

$$
\left(I_{a}^{\alpha} f\right)(t)=\frac{1}{\Gamma(\alpha)} \int_{a}^{t}(t-s)^{\alpha-1} f(s) d s, \quad \text { a.e. } a \leq t \leq b,
$$

where $\Gamma$ denotes the gamma function.

Definition 2 Let $\alpha>0$ and $n=[\alpha]+1$, where $[\alpha]$ denotes the integer part of $\alpha$. The Riemann-Liouville fractional integral of order $\alpha$ of $f:[a, b] \rightarrow \mathbb{R}$ is defined as

$$
\left(D_{a}^{\alpha} f\right)(t)=\left(\frac{d}{d t}\right)^{n}\left(I_{a}^{n-\alpha} f\right)(t)=\frac{1}{\Gamma(n-\alpha)} \frac{d^{n}}{d t^{n}} \int_{a}^{t} \frac{f(s)}{(t-s)^{\alpha-n+1}} d s, \quad \text { a.e. } a \leq t \leq b,
$$

provided that the right-hand side is defined almost everywhere in $[a, b]$.

Let $\alpha>0$ and $n=[\alpha]+1$. We denote by $A C^{\alpha}([a, b])$ the set of functions $f:[a, b] \rightarrow \mathbb{R}$ satisfying

$$
f(t)=\sum_{i=0}^{n-1} \frac{c_{i}}{\Gamma(\alpha-n+1+i)}(t-a)^{\alpha-n+i}+\left(I_{a}^{\alpha} \theta\right)(t), \quad \text { a.e. } a \leq t \leq b,
$$

where $c_{i}, i=0, \ldots, n-1$, are constants and $\theta \in L^{1}(a, b)$.

Lemma 3 (see [17]) Let $\alpha>0, n=[\alpha]+1$ and $f \in L^{1}(a, b)$. Then $D_{a}^{\alpha} f$ exists almost everywhere in $[a, b]$ if and only if $f \in A C^{\alpha}([a, b])$. In this case, one has

$$
\left(D_{a}^{\alpha} f\right)(t)=\theta(t), \quad \text { a.e. } a \leq t \leq b .
$$

Lemma 4 (see [20]) Let $\alpha>0$ and $n=[\alpha]+1 \geq 2$. Let $v \in C([a, b]) \cap A C^{\alpha}([a, b])$ be such that

$$
\left(D_{a}^{\alpha} v\right)(t)=y(t), \quad a<t<b,
$$

where $y \in C([a, b])$. Then $c_{0}=v(a)=0$, where $c_{0}$ is the constant appearing in (1.6).

Lemma 5 (see [24]) Let $\alpha, \beta>0$ and $f \in L^{1}(a, b)$. Then

(i) $I_{a}^{\alpha}\left(I_{a}^{\beta} f\right)(t)=\left(I_{a}^{\alpha+\beta} f\right)(t)$, a.e. $a \leq t \leq b$.

(ii) $D_{a}^{\alpha}\left(I_{a}^{\alpha} f\right)(t)=f(t)$, a.e. $a \leq t \leq b$. 


\section{Main results}

We start this section by deriving the Green's function for Problem (1.5). In the particular case $a=0$ and $b=1$, the Green's function was obtained in [13].

Lemma 6 Let $n \geq 3, n-1<v<n, n-3<\alpha<n-2$ and $y \in C([a, b])$. Then the fractional boundary value problem

$$
\left\{\begin{array}{l}
D_{a}^{v} x(t)+y(t)=0, \quad a<t<b, \\
x^{(i)}(a)=0, \quad 0 \leq i \leq n-2, \quad\left[D_{a}^{\alpha} x(t)\right]_{t=b}=0
\end{array}\right.
$$

admits a unique solution $x \in C([a, b]) \cap A C^{v}([a, b])$, which is given by

$$
x(t)=\int_{a}^{b} G(t, s) y(s) d s, \quad a \leq t \leq b,
$$

where the Green's function $G(t, s)$ is given by

$$
G(t, s)=\frac{1}{\Gamma(\nu)}\left\{\begin{array}{l}
\frac{(t-a)^{\nu-1}(b-s)^{\nu-\alpha-1}}{(b-a) \nu-1}-(t-s)^{\nu-1}, \quad a \leq s \leq t \leq b, \\
\frac{(t-a)^{\nu-1}(b-s)^{\nu-\alpha-1}}{(b-a)^{\nu-\alpha-1}}, \quad a \leq t \leq s \leq b .
\end{array}\right.
$$

Proof By Lemmas 3 and 4, one has

$$
x(t)=d_{1}(t-a)^{\nu-1}+d_{2}(t-a)^{\nu-2}+\cdots+d_{n-1}(t-a)^{\nu-n+1}-I_{a}^{\nu} y(t), \quad a \leq t \leq b,
$$

for some constants $d_{i} \in \mathbb{R}, i=1,2, \ldots, n-1$. Using the boundary conditions $x^{(i)}(a)=0$, $1 \leq i \leq n-2$, one obtains

$$
d_{2}=d_{3}=\cdots=d_{n-1}=0,
$$

which yields

$$
x(t)=d_{1}(t-a)^{\nu-1}-I_{a}^{v} y(t) .
$$

Next, taking into account that

$$
D_{a}^{\alpha}\left[(t-a)^{\nu-1}\right]=\frac{\Gamma(v)}{\Gamma(v-\alpha)}(t-a)^{\nu-\alpha-1}
$$

and that (see (i) and (ii) of Lemma 5)

$$
D_{a}^{\alpha} I_{a}^{v} y(t)=D_{a}^{\alpha} I_{a}^{\alpha}\left(I_{a}^{\nu-\alpha} y\right)(t)=\left(I_{a}^{\nu-\alpha} y\right)(t),
$$

one obtains

$$
D_{a}^{\alpha} x(t)=\frac{d_{1} \Gamma(v)}{\Gamma(v-\alpha)}(t-a)^{\nu-\alpha-1}-I_{a}^{\nu-\alpha} y(t),
$$


i.e.,

$$
D_{a}^{\alpha} x(t)=\frac{d_{1} \Gamma(v)}{\Gamma(\nu-\alpha)}(t-a)^{\nu-\alpha-1}-\frac{1}{\Gamma(v-\alpha)} \int_{a}^{t}(t-s)^{\nu-\alpha-1} y(s) d s .
$$

The above equality and the boundary condition $\left[D_{a}^{\alpha} x(t)\right]_{t=b}=0$ yield

$$
\frac{c_{1} \Gamma(v)}{\Gamma(v-\alpha)}(b-a)^{\nu-\alpha-1}-\frac{1}{\Gamma(v-\alpha)} \int_{a}^{b}(b-s)^{\nu-\alpha-1} y(s) d s=0,
$$

which implies that

$$
d_{1}=\frac{1}{\Gamma(\nu)(b-a)^{\nu-\alpha-1}} \int_{a}^{b}(b-s)^{\nu-\alpha-1} y(s) d s .
$$

Therefore,

$$
x(t)=\frac{(t-a)^{\nu-1}}{\Gamma(v)(b-a)^{\nu-\alpha-1}} \int_{a}^{b}(b-s)^{\nu-\alpha-1} y(s) d s-\frac{1}{\Gamma(\nu)} \int_{a}^{t}(t-s)^{\nu-1} y(s) d s
$$

or, equivalently,

$$
x(t)=\int_{a}^{b}\left(\frac{(t-a)^{\nu-1}(b-s)^{\nu-\alpha-1}}{\Gamma(v)(b-a)^{\nu-\alpha-1}}-\frac{(t-s)^{\nu-1}}{\Gamma(v)} \chi_{[a, t]}(s)\right) y(s) d s,
$$

where $\chi$ denotes the characteristic function. Therefore, the result follows.

The following lemma provides some useful properties of the Green's function $G(t, s)$.

Lemma 7 The Green's function $G(t, s)$ satisfies the following properties:

(i) $G$ is a continuous function in $[a, b] \times[a, b]$.

(ii) $G(t, s) \geq 0$, for every $(t, s) \in[a, b] \times[a, b]$.

(iii) $G$ is non-decreasing with respect to the first variable.

Proof (i) is obvious. It is clear that, for $a \leq t \leq s \leq b$, we have

$$
\Gamma(v) G(t, s)=\frac{(t-a)^{\nu-1}(b-s)^{\nu-\alpha-1}}{(b-a)^{\nu-\alpha-1}} \geq 0 .
$$

On the other hand, for $a \leq s<t \leq b$, we have

$$
\begin{aligned}
\Gamma(v) G(t, s) & =\frac{(t-a)^{\nu-1}(b-s)^{\nu-\alpha-1}}{(b-a)^{\nu-\alpha-1}}-(t-s)^{\nu-1} \\
& =(t-a)^{\nu-1}\left(\frac{(b-a)-(s-a)}{b-a}\right)^{\nu-\alpha-1}-((t-a)-(s-a))^{\nu-1} \\
& =(t-a)^{\nu-1}\left(1-\frac{s-a}{b-a}\right)^{\nu-\alpha-1}-(t-a)^{\nu-1}\left(1-\frac{s-a}{t-a}\right)^{\nu-1} \\
& =(t-a)^{\nu-1}\left[\left(1-\frac{s-a}{b-a}\right)^{\nu-\alpha-1}-\left(1-\frac{s-a}{t-a}\right)^{\nu-1}\right]
\end{aligned}
$$




$$
\begin{aligned}
& \geq(t-a)^{\nu-1}\left[\left(1-\frac{s-a}{b-a}\right)^{\nu-\alpha-1}-\left(1-\frac{s-a}{b-a}\right)^{\nu-1}\right] \\
& =(t-a)^{\nu-1}\left(1-\frac{s-a}{b-a}\right)^{\nu-1}\left[\left(1-\frac{s-a}{b-a}\right)^{-\alpha}-1\right] \\
& \geq 0 .
\end{aligned}
$$

Therefore, (ii) follows. In order to prove (iii), we shall study the sign of $\partial_{t} G$; the partial derivative of the function $G$ with respect to the first variable $t$. For $a \leq s<t \leq b$, we have

$$
\begin{aligned}
\Gamma(\nu) \frac{\partial G(t, s)}{\partial t} & =\frac{(\nu-1)(t-a)^{\nu-2}(b-s)^{\nu-\alpha-1}}{(b-a)^{\nu-\alpha-1}}-(v-1)(t-s)^{\nu-2} \\
& =(\nu-1)(t-a)^{\nu-2}\left(\frac{(b-a)-(s-a)}{b-a}\right)^{\nu-\alpha-1}-(v-1)((t-a)-(s-a))^{\nu-2} \\
& =(v-1)(t-a)^{\nu-2}\left[\left(1-\frac{s-a}{b-a}\right)^{\nu-\alpha-1}-\left(1-\frac{s-a}{t-a}\right)^{\nu-2}\right] \\
& \geq(v-1)(t-a)^{\nu-2}\left[\left(1-\frac{s-a}{b-a}\right)^{\nu-\alpha-1}-\left(1-\frac{s-a}{b-a}\right)^{\nu-2}\right] \\
& =(v-1)(t-a)^{\nu-2}\left(1-\frac{s-a}{b-a}\right)^{\nu-2}\left[\left(1-\frac{s-a}{b-a}\right)^{1-\alpha}-1\right] \\
& \geq 0 .
\end{aligned}
$$

On the other hand, for $a \leq t \leq s \leq b$, we have

$$
\Gamma(\nu) \frac{\partial G(t, s)}{\partial t}=\frac{(\nu-1)(t-a)^{\nu-2}(b-s)^{\nu-\alpha-1}}{(b-a)^{\nu-\alpha-1}} \geq 0 .
$$

This proves the non-decreasing character of the function $G$ with respect to its first variable.

The following result is an immediate consequence of Lemma 7.

\section{Lemma 8 The Green's function G satisfies}

$$
0 \leq G(a, s) \leq G(t, s) \leq G(b, s)=\max _{\tau \in[a, b]} G(\tau, s), \quad(t, s) \in[a, b] \times[a, b] .
$$

Our first result in this paper is the following Hartman-Wintner type inequality.

Theorem 9 Suppose that (1.5) has a nontrivial continuous solution. Then

$$
\int_{a}^{b}(b-s)^{\nu-1}\left[\left(\frac{b-a}{b-s}\right)^{\alpha}-1\right]|q(s)| d s \geq \Gamma(\nu) .
$$

Proof We endow the space $C([a, b])$ with the Chebyshev norm

$$
\|u\|_{\infty}=\max \{|u(t)|: a \leq t \leq b\}, \quad u \in C([a, b] . \mathbb{R}) .
$$


Let $x \in C([a, b])$ be a nontrivial solution to $(1.5)\left(\|x\|_{\infty} \neq 0\right)$. By Lemma 6 , we have

$$
x(t)=\int_{a}^{b} G(t, s) q(s) x(s) d s, \quad t \in[a, b]
$$

which yields

$$
\|x\|_{\infty} \leq\|x\|_{\infty} \int_{a}^{b}|G(t, s) \| q(s)| d s .
$$

Since $x$ is nontrivial, we have

$$
1 \leq \int_{a}^{b}|G(t, s)||q(s)| d s .
$$

Taking into account Lemma 8, we obtain

$$
\begin{aligned}
1 & \leq \int_{a}^{b} G(b, s)|q(s)| d s \\
& =\frac{1}{\Gamma(\nu)} \int_{a}^{b}\left[\frac{(b-a)^{\nu-1}(b-s)^{\nu-\alpha-1}}{(b-a)^{\nu-\alpha-1}}-(b-s)^{\nu-1}\right]|q(s)| d s \\
& =\frac{1}{\Gamma(\nu)} \int_{a}^{b}(b-s)^{\nu-1}\left[\left(\frac{b-a}{b-s}\right)^{\alpha}-1\right]|q(s)| d s,
\end{aligned}
$$

which yields the desired inequality.

In what follows, we present a Lyapunov type inequality associated to Problem (1.5).

Consider the function

$$
\varphi(s)=(b-a)^{\alpha}(b-s)^{\nu-\alpha-1}-(b-s)^{\nu-1}, \quad a \leq s \leq b .
$$

We have

$$
\begin{aligned}
\varphi^{\prime}(s) & =-(v-\alpha-1)(b-a)^{\alpha}(b-s)^{\nu-\alpha-2}+(v-1)(b-s)^{\nu-2} \\
& =(b-s)^{\nu-\alpha-2}\left[-(v-\alpha-1)(b-a)^{\alpha}+(v-1)(b-s)^{\alpha}\right] .
\end{aligned}
$$

One observes easily that

$$
\varphi^{\prime}(s)=0, \quad s \in[a, b] \Longleftrightarrow s \in\left\{b, s^{*}\right\},
$$

where

$$
s^{*}=b-\left(\frac{v-\alpha-1}{v-1}\right)^{1 / \alpha}(b-a) .
$$

Note that, from the assumptions $n \geq 3, n-1<v<n$ and $n-3<\alpha<n-2$, one has $s^{*} \in(a, b)$. Moreover, it is easy to check that

$$
\varphi^{\prime}(s) \geq 0, \quad s \in\left[a, s^{*}\right]
$$


and

$$
\varphi^{\prime}(s)<0, \quad s \in\left(s^{*}, b\right) .
$$

Therefore, since $\varphi(b)=0$, we deduce that

$$
\varphi\left(s^{*}\right)=\max \{\varphi(s): a \leq s \leq b\} .
$$

A simple computation yields

$$
\varphi\left(s^{*}\right)=\frac{\alpha(b-a)^{\nu-1}}{v-1}\left(\frac{v-\alpha-1}{v-1}\right)^{\frac{v-\alpha-1}{\alpha}} .
$$

Now, under the assumptions of Theorem 9, one has

$$
\begin{aligned}
\Gamma(\nu) & \leq \int_{a}^{b}(b-s)^{v-1}\left[\left(\frac{b-a}{b-s}\right)^{\alpha}-1\right]|q(s)| d s \\
& =\int_{a}^{b} \varphi(s)|q(s)| d s \\
& \leq \int_{a}^{b} \varphi\left(s^{*}\right)|q(s)| d s \\
& =\frac{\alpha(b-a)^{v-1}}{v-1}\left(\frac{v-\alpha-1}{v-1}\right)^{\frac{v-\alpha-1}{\alpha}} \int_{a}^{b}|q(s)| d s .
\end{aligned}
$$

Therefore, one obtains the following Lyapunov type inequality for Problem (1.5).

Corollary 10 Suppose that (1.5) has a nontrivial continuous solution. Then

$$
\int_{a}^{b}|q(s)| d s \geq\left(\frac{v-1}{\alpha}\right)\left(\frac{v-1}{v-\alpha-1}\right)^{\frac{v-\alpha-1}{\alpha}} \frac{\Gamma(v)}{(b-a)^{v-1}} .
$$

Theorem 9 and Corollary 10 have as consequences some Hartman-Wintner and Lyapunov type inequalities for ordinary boundary value problems. Indeed, in the limit cases $\nu \rightarrow n^{-}$and $\alpha \rightarrow(n-2)^{-},(1.5)$ reduces (formally) to the ordinary boundary value problem

$$
\left\{\begin{array}{l}
x^{(n)}(t)+q(t) x(t)=0, \quad a<t<b \\
x(a)=x^{\prime}(a)=\cdots=x^{(n-2)}(a)=x^{(n-2)}(b)=0
\end{array}\right.
$$

where $n \in \mathbb{N}, n \geq 3$, and $q \in C([a, b])$. Hence, one deduces the following results.

Corollary 11 Suppose that (2.1) has a nontrivial solution. Then

$$
\int_{a}^{b}(b-s)^{n-1}\left[\left(\frac{b-a}{b-s}\right)^{n-2}-1\right]|q(s)| d s \geq(n-1) !
$$

Corollary 12 Suppose that (2.1) has a nontrivial solution. Then

$$
\int_{a}^{b}|q(s)| d s \geq \frac{(n-1)^{\frac{2 n-3}{n-2}}(n-3) !}{(b-a)^{n-1}}
$$


Finally, we present a numerical example where our results can be applied. As we mentioned at Introduction, this example describes the deflection or deformation of an elastic beam under a determined force.

Example 13 Consider the following boundary value problem:

$$
\left\{\begin{array}{l}
x^{(4)}(t)+q(t) x(t)=0, \quad 0<t<1 \\
x(0)=x^{\prime}(0)=x^{\prime \prime}(0)=x^{\prime \prime}(1)=0
\end{array}\right.
$$

where $q:[0,1] \rightarrow \mathbb{R}$ is a continuous function. If

$$
\int_{0}^{1}|q(s)| d s<9 \sqrt{3}
$$

then (2.2) admits no nontrivial solutions. Indeed, if $x$ is a nontrivial solution to (2.2), then, by Corollary 12 with $n=4$, one obtains

$$
\int_{0}^{1}|q(s)| d s \geq 3^{5 / 2}=9 \sqrt{3}
$$

which contradicts (2.3).

Particularly, the boundary value problem

$$
\left\{\begin{array}{l}
x^{(4}(t)+\ln (1+t) x(t)=0, \quad 0<t<1, \\
x(0)=x^{\prime}(0)=x^{\prime \prime}(0)=x^{\prime \prime}(1)=0,
\end{array}\right.
$$

has the trivial solution as unique solution, since

$$
\int_{0}^{1} \ln (1+s) d s=2 \ln 2-1<9 \sqrt{3}
$$

\section{Application}

In this section, we present some applications of the previous obtained inequalities to eigenvalue problems.

We say that $\lambda \in \mathbb{R}$ is an eigenvalue of the fractional boundary value problem

$$
\left\{\begin{array}{l}
-D_{a}^{v} x(t)=\lambda x(t), \quad a<t<b, \\
x^{(i)}(a)=0, \quad 0 \leq i \leq n-2, \quad\left[D_{a}^{\alpha} x(t)\right]_{t=b}=0
\end{array}\right.
$$

where $n \geq 3, n-1<v<n$ and $n-3<\alpha<n-2$, if Problem (3.1) admits at least a nontrivial continuous solution $x_{\lambda}$, which is called an eigenvector associated to the eigenvalue $\lambda$.

Corollary 14 If $\lambda$ is an eigenvalue of Problem (3.1), then

$$
|\lambda| \geq \frac{v(v-\alpha) \Gamma(v)}{\alpha(b-a)^{v}}
$$


Proof Let $\lambda$ be an eigenvalue of Problem (3.1). Then Problem (3.1) admits a nontrivial solution $x_{\lambda} \in C([a, b])$. By Theorem 9, one has

$$
|\lambda| \int_{a}^{b}(b-s)^{v-1}\left[\left(\frac{b-a}{b-s}\right)^{\alpha}-1\right] d s \geq \Gamma(v) .
$$

An elementary calculation yields

$$
\int_{a}^{b}(b-s)^{\nu-1}\left[\left(\frac{b-a}{b-s}\right)^{\alpha}-1\right] d s=\frac{\alpha}{v(v-\alpha)}(b-a)^{\nu} .
$$

Therefore,

$$
\frac{\alpha}{v(v-\alpha)}(b-a)^{v}|\lambda| \geq \Gamma(v)
$$

which yields (3.2).

Corollary 15 If $\lambda$ is an eigenvalue of the ordinary boundary value problem

$$
\left\{\begin{array}{l}
-x^{(n)}(t)=\lambda x(t), \quad a<t<b, \\
x(a)=x^{\prime}(a)=\cdots=x^{(n-2)}(a)=x^{(n-2)}(b)=0,
\end{array}\right.
$$

where $n \in \mathbb{N}, n \geq 3$, then

$$
|\lambda| \geq \frac{2 n(n-1)(n-3) !}{(b-a)^{n}} .
$$

Proof Passing to the limits as $v \rightarrow n^{-}$and $\alpha \rightarrow(n-2)^{-}$in (3.2), the desired inequality follows.

\section{Conclusion}

Some Hartman-Wintner type inequalities are established for a given higher order fractional boundary value problem. Such inequalities are useful in many applications related to the study of different properties of the solutions. The approach used in this paper is based on the calculation of the Green's function associated to the considered problem and the computation of its maximum. On the other hand, in some cases, finding the maximum of the Green's function is not an easy task. In the case of integer order derivatives, variational methods can be used in order to avoid such problem (see, for example [7]). In the fractional case, due to the nonlocal properties of the fractional derivative, some problems arise using variational methods (integration by parts, Leibniz's rule, ...). Therefore, other approaches must be pursued in order to study fractional boundary value problems.

\section{Acknowledgements}

The first and second authors were partially supported by the project MTM2016-79436-P. The third author is supported by Researchers Supporting Project RSP-2019/4, King Saud University, Saudi Arabia, Riyadh. 


\section{Competing interests}

The authors declare that they have no competing interests.

\section{Authors' contributions}

All authors jointly worked on the results and they read and approved the final manuscript.

\section{Author details}

'Departamento de Matemáticas, Universidad de Las Palmas de Gran Canaria, Las Palmas de Gran Canaria, Spain.

${ }^{2}$ Department of Mathematics, College of Science, King Saud University, Riyadh, Saudi Arabia.

\section{Publisher's Note}

Springer Nature remains neutral with regard to jurisdictional claims in published maps and institutional affiliations.

\section{Received: 9 August 2019 Accepted: 23 October 2019 Published online: 01 November 2019}

\section{References}

1. Ahmad, B., Nieto, J.J: Existence results for a coupled system of nonlinear fractional differential equations with three-point boundary conditions. Comput. Math. Appl. 58, 1838-1843 (2009)

2. Ahmad, B., Nieto, J.J.: Boundary value problems for a class of sequential integrodifferential equations of fractional order. J. Funct. Spaces Appl. 2013, Article ID 149659 (2013)

3. Ahmed, E., El-Saka, H.A.: On fractional order models for hepatitis C. Nonlinear Biomed. Phys. 4, 1 (2010)

4. Arafa, A.A.M., Rida, S.Z., Khalil, M.: Fractional modeling dynamics of HIV and CD+ T-cells during primary infection. Nonlinear Biomed. Phys. 6, 1 (2012)

5. Brown, R.C., Hinton, D.B.: Opial's inequality and oscillation of 2 nd order equations. Proc. Am. Math. Soc. 125 , 1123-1129 (1997)

6. Cabrera, I., Sadarangani, K., Samet, B.: Hartman-Wintner-type inequalities for a class of nonlocal fractional boundary value problems. Math. Methods Appl. Sci. 40(1), 129-136 (2017)

7. Çakmak, D.: Lyapunov-type integral inequalities for certain higher order differential equations. Appl. Math. Comput. 216, 368-373 (2010)

8. Chalishajar, D.N., Karthikeyan, K.: Existence and uniqueness results for boundary value problems of higher order fractional integro-differential equations involving Gronwall's inequality in Banach spaces. Acta Math. Sci. 33, 758-772 (2013)

9. Chalishajar, D.N., Karthikeyan, K.: Boundary value problems for impulsive fractional evolution integrodifferential equations with Gronwall's inequality in Banach spaces. Discontin. Nonlinearity Complex. 3, 33-48 (2014)

10. Cheng, S.S.: A discrete analogue of the inequality of Lyapunov. Hokkaido Math. J. 12, 105-112 (1983)

11. Ferreira, R.A.C.: A Lyapunov-type inequality for a fractional boundary value problem. Fract. Calc. Appl. Anal. 16(4), 978-984 (2013)

12. Ferreira, R.A.C.: On a Lyapunov-type inequality and the zeros of a certain Mittag-Leffler function. J. Math. Anal. Appl. 412(2), 1058-1063 (2014)

13. Goodrich, C.S.: Existence of a positive solution to a class of fractional differential equations. Appl. Math. Lett. 23, 1050-1055 (2010)

14. Guseinov, G., Kaymakçalan, B.: Lyapunov inequalities for discrete linear Hamiltonian system. Comput. Math. Appl. 45, 1399-1416 (2003)

15. Hartman, P., Wintner, A.: On an oscillation criterion of Liapounoff. Am. J. Math. 73, 885-890 (1951)

16. Hilfer, R: Applications of Fractional Calculus in Physics. World Scientific, Singapore (2000)

17. Idczak, D., Walczak, S.: Fractional Sobolev spaces via Riemann-Liouville derivatives. J. Funct. Spaces Appl. 2013, Article ID 128043 (2013)

18. Jesus, I., Machado, J., Cunha, J.: Fractional electrical impedances in botanical elements. J. Vib. Control 14, 1389-1402 (2008)

19. Jiang, L., Zhou, Z:: Lyapunov inequality for linear Hamiltonian systems on time scales. J. Math. Anal. Appl. 310 , 579-593 (2005)

20. Jleli, M., Nieto, J.J., Samet, B.: Lyapunov-type inequalities for a higher order fractional differential equation with fractional integral boundary conditions. Electron. J. Qual. Theory Differ. Equ. 2017, 16 (2017)

21. Jeli, M., Samet, B.: Lyapunov-type inequalities for a fractional differential equation with mixed boundary conditions. Math. Inequal. Appl. 18(2), 443-451 (2015)

22. Jleli, M., Samet, B.: Lyapunov-type inequalities for fractional boundary value problems. Electron. J. Differ. Equ. 2015, 88 (2015)

23. Jeli, M., Samet, B.: A Lyapunov-type inequality for a fractional q-difference boundary value problem. J. Nonlinear Sci. Appl. 9, 1965-1976 (2016)

24. Kilbas, A.A., Srivastava, H.M., Trujillo, J.J.: Theory and Applications of Fractional Differential Equations. North-Holland Mathematics Studies, vol. 204. Elsevier, Amsterdam (2006)

25. Liang, S., Zhang, J.: Positive solutions for boundary value problems of nonlinear fractional differential equations. Nonlinear Anal. 71, 5545-5550 (2009)

26. Liapunov, A.M.: Problème général de la stabilité du mouvement. Ann. Fac. Sci. Univ. Toulouse 2, 203-407 (1907)

27. Nieto, J.J., Pimentel, J.: Positive solutions of a fractional thermostat model. Bound. Value Probl. 2013, 5 (2013)

28. O'Regan, D., Samet, B.: Lyapunov-type inequalities for a class of fractional differential equations. J. Inequal. Appl. 2015 247 (2015)

29. Pachpatte, B.G.: On Lyapunov-type inequalities for certain higher order differential equations. J. Math. Anal. Appl. 195, 527-536 (1995)

30. Parhi, N., Panigrahi, S.: On Liapunov-type inequality for third-order differential equations. J. Math. Anal. Appl. 233(2), 445-460 (1999) 
31. Podlubny, I: Geometric and physical interpretation of fractional integration and fractional differentiation. Fract. Calc. Appl. Anal. 5, 367-386 (2002)

32. Rong, J., Bai, C.: Lyapunov-type inequality for a fractional differential equation with fractional boundary conditions. Adv. Differ. Equ. 2015, 82 (2015)

33. Samko, S.G., Kilbas, A.A., Marichev, O.I.: Fractional Integrals and Derivatives. Gordon and Breach, Yverdon (1993) (translated from the 1987 Russian original)

34. Yang, X., Lo, K.: Lyapunov-type inequality for a class of even-order differential equations. Appl. Math. Comput. 215, 3884-3890 (2010)

Submit your manuscript to a SpringerOpen ${ }^{\mathcal{O}}$ journal and benefit from:

- Convenient online submission

$\rightarrow$ Rigorous peer review

Open access: articles freely available online

- High visibility within the field

- Retaining the copyright to your article

Submit your next manuscript at $\boldsymbol{\Delta}$ springeropen.com 